\title{
Efficacy of Pulsed Low-Frequency Magnetic Field Therapy on Patients with Chronic Low Back Pain: A Randomized Double-Blind Placebo-Controlled Trial
}

\author{
Khalid Abdulkareem Alzayed ${ }^{1}$, Saad Mohammed Alsaadi ${ }^{2}$ \\ ${ }^{1}$ Musculoskeletal Physical Therapy, Imam Abdulrahman Bin Faisal University, Dammam, Saudi Arabia \\ ${ }^{2}$ Musculoskeletal Division, Department of Physical Therapy, College of Applied Medical Sciences, Imam Abdulrahman Bin Faisal University, \\ Dammam, Saudi Arabia
}

Study Design: A randomized double-blind placebo-controlled trial.

Purpose: To investigate the efficacy of pulsed electromagnetic field (PEMF) therapy combined with therapeutic exercises in the treatment of chronic low back pain (CLBP).

Overview of Literature: Low back pain (LBP) is the most common musculoskeletal pain disorder. Most available interventions for CLBP have modestly beneficial outcomes. Despite the potential effect of PEMF therapy on LBP, there have been few studies regarding its effectiveness.

Methods: Forty-two patients (22 males, 20 females), were randomized into either the treatment group (PEMF and therapeutic exercises) or placebo group (sham PEMF and exercises). Primary outcome measures were pain intensity on the 10-point Numeric PainRating Scale and disability measured by the Roland-Morris Disability Questionnaire. The patients were assessed at baseline, during the treatment period (weeks 3, 6, and 9), and after treatment (week 13).

Results: The treatment group experienced a more rapid improvement in both pain and disability compared with the placebo group. The analysis showed a significant improvement in the pain intensity and disability scores in the treatment group at week $3(p<0.05)$, whereas an improvement in the placebo group was detected at week 6 . The significant improvement in both groups was sustained for weeks 6,9 , and 13. There was no difference between the groups in scores of pain intensity and disability at weeks 6 and 13 .

Conclusions: PEMF therapy improved pain and disability in patients with CLBP. However, it does not seem to be superior to other treatment options.

Keywords: Lower back pain; Magnetic field therapy

\section{Introduction}

Low back pain (LBP) is the most common musculoskeletal pain disorder that causes primary care visits in the United States [1]. LBP is a highly prevalent condition and affects more than $80 \%$ of the population at some point in their lifetime [1]. Chronic low back pain (CLBP), defined as any pain or discomfort between the 12th rib and gluteal crest persisting for more than 12 weeks [2], is one of the leading causes of disability globally [3]. Emerging

Received Feb 8, 2019; Revised Mar 23, 2019; Accepted May 3, 2019

Corresponding author: Khalid Abdulkareem Alzayed

Physical Therapy Department, King Fahad Specialist Hospital, Dammam 31444, Saudi Arabia

Tel: +966-138442222 (Ext: 1930), E-mail: Khalid.Zayed@kfsh.med.sa 
evidence suggests that approximately $33 \%$ of acute LBP patients fail to recover and develop CLBP [4]. There is evidence that CLBP is often associated with a significant economic burden, due to restricted physical ability, career burden, activity limitation, work absenteeism, and cost of medical care [4].

There are various guidelines for the management of CLBP, including exercise, acupuncture, massage therapy, yoga, cognitive behavioral therapy, progressive relaxation, spinal manipulation, and interdisciplinary rehabilitation [5]. However, research shows that these interventions only have modestly beneficial outcomes [6]. Pulsed electromagnetic field (PEMF) therapy has been used to manage various pain conditions; it aims to influence behavioral as well as physiological parameters in order to alleviate pain. However, its efficacy in treating CLBP has not been well established. Moreover, very few studies have been conducted on PEMF and CLBP, and the findings of these studies are inconsistent. Finegold and Flamm [7] found that PEMF therapy had little effect on the pain intensity among patients with CLBP. In contrast, Loo [8] in 2009 and Arneja et al. [9] in 2016 reported that the use of PEMF positively contributed to patient recovery. The conflicting findings seem to be attributed to study differences in sample size, research parameters, and treatment duration.

This study primarily investigated the efficacy of PEMF therapy on the reduction of pain intensity and functional disability among patients with CLBP. Secondary outcomes of interest were its effect on psychological outcomes (depression, anxiety, and stress), sleep patterns, and patientperceived effects. We hypothesized that PEMF reduces the intensity of pain associated with CLBP and improves functional ability, psychological aspects, and sleep patterns.

\section{Materials and Methods}

\section{Study design}

The study was designed as a randomized double-blind placebo-controlled trial. The study participants were patients with CLBP referred to the physical therapy department at the King Fahad Specialist Hospital in Dammam, Saudi Arabia (KFSH-D). We calculated that the total patient sample size required was 40 in order to have a $90 \%$ chance of detecting differences that were significant at the 5\% alpha level [10]. The sample size was increased by $5 \%$ to compensate for patient dropout from the study. The total sample size was 52 patients, whereas the number of initial participants per group was 26 patients; 10 patients did not complete the treatment sessions, and 42 were included in the final analysis (20 in the treatment group, 22 in the control group). The study inclusion criteria were males or females aged 18-60 years presenting with a primary complaint of back pain rated $\geq 5$ on the 10-point Numeric Pain-Rating Scale (NPRS) in the area between the 12th rib and the iliac crease for more than 12 weeks, with or without leg pain; other inclusion criteria included the ability to read, understand, and follow the study instructions. Patients with CLBP were excluded if they had known or suspected serious spinal pathology (e.g., metastatic, inflammatory, or infectious diseases of the spine; cauda equina syndrome; or spinal fracture), took blood-thinning or clotting inhibitor medication, or had spinal surgery within the preceding 6 months. Additionally, patients with an existing or planned pregnancy during the study period, a history of epilepsy or psychosis, immunosuppression (primary immunodeficiency, condition requiring immunosuppressive medication, inherited disease affecting the immune system, and neutropenia), and implanted metal devices (pacemaker, defibrillator, neurostimulator, spinal cord stimulator, bone stimulator, cochlear implant, and others) were also excluded.

The sample was divided into two groups (treatment group and control group) through stratified random sampling using computer-generated block randomization. The treatment group received PEMF therapy via a BioElectro-Magnetic-Energy-Regulation (BEMER) device, whereas the control group received sham PEMF therapy (the BEMER device was not active during the treatment session). Both groups received exercise therapy. Patients underwent PEMF or sham PEMF therapy for 3 months (13 weeks) for a total of 39 sessions (3-5 times per week) administered for 20 minutes per session. The outcome measures were assessed at the first session, at weeks 3, 6, and 9 during treatment, and then at week 13, posttreatment. The participants, researchers, and assessors were blinded to the use of PEMF.

\section{Outcome measures}

1) Pain intensity

Pain intensity was measured using the 10-point NPRS, 
with 0 representing "no pain" and 10 representing "worst imaginable" pain. This scale has been validated for use in the measurement of pain intensity among patients with obvious pain [11]; the Arabic version of the NPRS has been previously validated and shown to be reliable and comparable with the English version [12].

\section{2) Physical disability}

The Roland-Morris Disability Questionnaire (RMDQ24) was used to evaluate self-rated physical disability due to LBP. It evaluates a patient's daily living aspects, such as sleeping, walking, lifting, resting, housework, appetite, dressing, and self-care, and has been shown to be a suitable measure for patients suffering from mild to moderate disability arising from acute, subacute, or chronic LBP [13]; the Arabic version of the RMDQ-24 has been reported to be valid and reliable [14].

\section{3) Sleep disturbance}

Item 6 of the Pittsburgh Sleep Quality Index (PSQI) was used to measure patients' quality and patterns of sleep over a 1-month period via a self-reported questionnaire and is presented in Table 1. The tool accurately detects sleep disturbance in patients with LBP [15], and the validity and reliability have been previously ascertained [16]; the Arabic version of the PSQI is also valid and reliable [17].

\section{4) Global perceived effect of change}

The global perceived effect (GPE) scale is widely used among patients with musculoskeletal disorders. It combines patient-perceived outcomes, such as mental health, physical role, social and physical functioning, health transition, emotional function, and general health, allowing the patient to integrate all factors to give an overall evaluation [18]. The scale consists of integers from -5 through 5 , with 0 representing "unchanged," 5 representing "completely recovered," and -5 representing "vastly worse."

5) Depression, anxiety, and stress

Depression, anxiety, and stress were evaluated using the
Depression Anxiety Stress Scale (DASS-21). The DASS-21 is self-administered and focuses on reports of low mood, motivation, and self-esteem; physiological arousal; perceived panic and fear; tension; and irritability. The subjects were asked to use a 4-point severity/frequency scale to rate the extent to which they had experienced each state over the previous week. Adequate construct validity and reliability have been ascertained [19]; the Arabic version has been validated and shown to be reliable [20].

\section{Interventions}

1) The BEMER device

The BEMER device administers PEMF noninvasively, operating using a weak magnetic field [21]. The therapy is based on the principle that charged molecules in human cells react with a magnetic field, resulting in enhanced biochemical reactions that eventually produce positive physiological effects, which include improved oxygen supply, enhanced circulation, activation of cellular metabolism, enhanced healing and cellular regeneration, increased removal of toxins, and improved general wellbeing and fitness [21]. The use of the BEMER device is not associated with any known side effects and poses no harm to the person operating it [21].

The BEMER device offers three predefined programs (P1-P3). P1 contains the low-intensity levels $2-4$ used for 4 minutes and is recommended for superficial areas with minor symptoms. P2 includes the middle-intensity levels 3-6 used for 16 minutes; it is recommended for a somewhat deeper area with moderate symptoms. P3 includes the strong intensity levels 6-10 used for 20 minutes, which is approved for deeper lying areas and for areas with severe symptoms. In the present study, the BEMER Pro Set device (BEMER International AG, Triesen, Liechtenstein), using the $\mathrm{P} 3$ program for 20 minutes with a mattress applicator (Body Pro) of average flux density 35 microtesla $(\mu \mathrm{T})$, was used (Fig. 1).

2) Study procedure and treatment protocol

We screened for conditions wherein consultation with

Table 1. The description of the Pittsburgh Sleep Quality Index-item 6

Item $\quad$ Very good (0) Fairly good (1) Fairly bad (2) Very bad (3)
During the past month, how often have you taken medicine (pre-
scribed or "over the counter") to help you sleep?




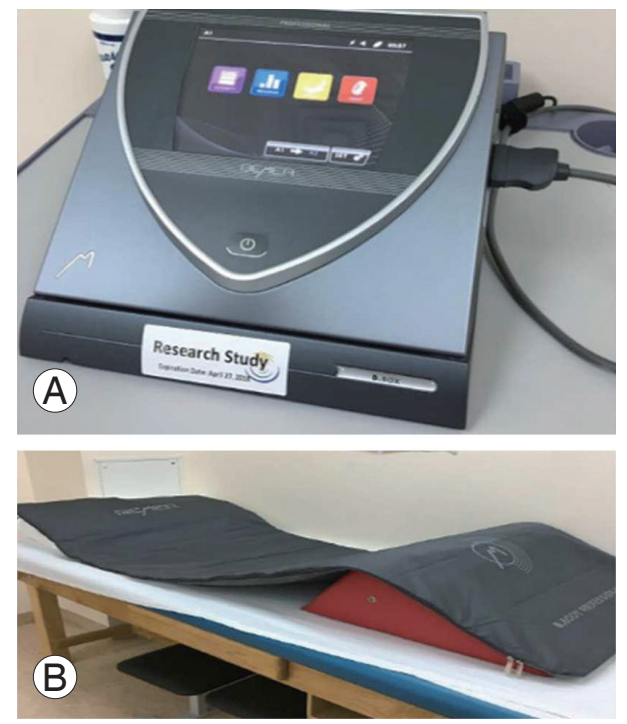

Fig. 1. (A) BEMER control unit. (B) Mattress applicator (Body Pro).

a medical doctor before the use of BEMER therapy is strongly recommended. This includes an unclear fever, infection, severe cardiac rhythm disorders, long-term use of $\beta$-receptor antagonists, long-term use of corticoid agents, and long-term use of coumarin derivatives. The patient was asked to lie down on his/her back on a full-body mat connected to a BEMER device placed over the treatment bed. The physical therapy technician started the BEMER device for 20 minutes and then switched off the device. The BEMER parameters were set and tested before the session. The device had no sound during the treatment session, and the sound could only be heard at the start of the setting when the participant and the principal investigator were out of the treatment area. The screen of the BEMER was covered with a pillowcase and facing the wall during the treatment session to ensure complete blindness of the participants. The first session lasted for an hour, during which the patient underwent a physical examination before the therapy session and primary sociodemographic data was collected. The participants were also trained how to record their pain intensity on the NPRS in their initial assessment. The NPRS was used to record the severity of the pain that the patient was undergoing and the progress of the pain, as the participants took the test regularly. However, if the patient's pain worsened during the study period, the treating physician would be notified and the patient would be advised to remain active and undergo non-pharmacological treatments, such as massage therapy and spinal manipulation $[5,22]$. Other treatment sessions were carried out on weeks 3, 6, 9, and 13 .

\section{3) Exercise therapy}

In addition to BEMER therapy, all participants in both groups received similar exercise programs, which were individually designed based on the physical therapist's assessment and included supervised stretching and strengthening. Exercise therapy is thought to improve pain and function in patients with CLBP $[22,23]$. Therapy consisted of self-stretching exercises for the lumbar erector spinae muscles and tissues posterior to the spine; mild stretching exercises of the hamstring, calf muscles, and back muscles for 30 seconds; and strengthening exercises for the back muscles (bridging and active back extensions) and abdominal muscles (sit-ups and posterior pelvic tilts). Each exercise was performed 3 times per session, holding for five counts with a 1 minute rest between each exercise.

\section{Statistical analysis}

Descriptive statistics were used to describe demographic data and group characteristics. A paired $t$-test was used to compare the differences before and after the interventions for both the treatment and control groups. An independent $t$-test was used to compare the two groups before the intervention, during the intervention (weeks 3, 6, and 9), and after the completion of the treatment (week 13). Statistical analysis was performed using IBM SPSS software for Windows ver. 20.0 (IBM Corp., Armonk, NY, USA). Statistical significance was defined as $p<0.05$.

\section{Ethical approval and informed consent}

Ethical approval from the Institutional Review Board at Imam Abdulrahman Bin Faisal University (IRB study no., IRB-PGS-2017-03-179) and KFSH-D (IRB study no., MED0330) was obtained prior to initiating the study. Informed consent was obtained from all participants.

\section{Results}

A total of 80 patients with CLBP were screened for eligibility, of which 28 did not meet the study criteria and were thus excluded. Ten of the remaining 52 eligible patients did not complete the treatment sessions. Finally, 42 patients were included for the analysis, of which 20 were allocated in the treatment group and 22 in the control group (Fig. 2). The baseline analysis of pain intensity and self-rated physical disability scores showed no statisti- 


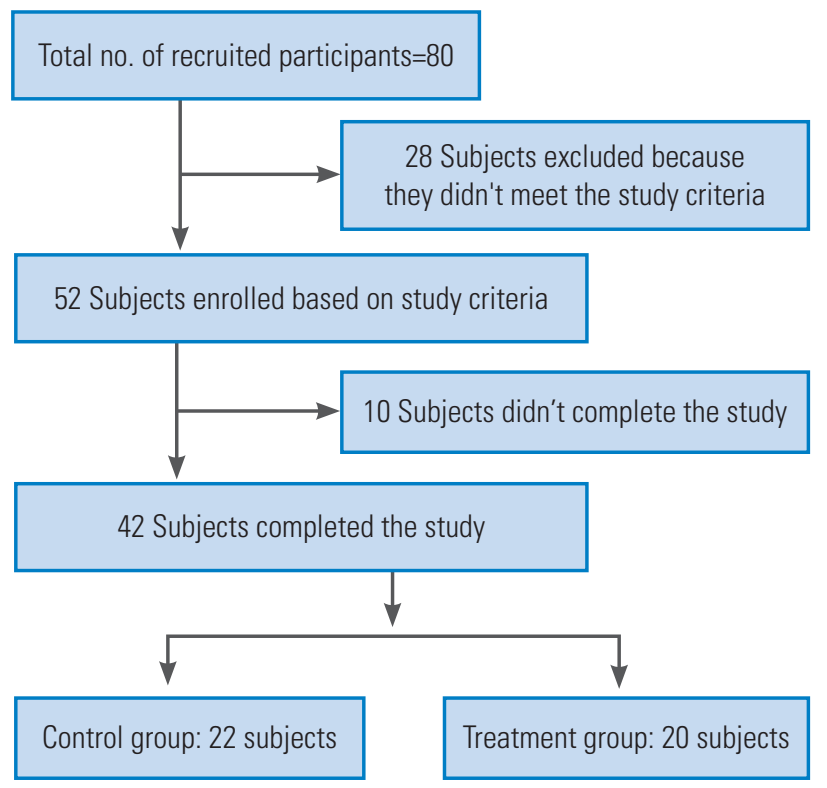

Fig. 2. A flow chart describing the patient recruitment process. cal difference between the treatment and control groups ( $p=0.87$ and 0.51 , respectively). In addition, the demographic characteristics also revealed no statistically significant difference between the groups in age, body mass index, marital status, gender, and duration of LBP. Importantly, there was no difference between the groups in pain medication usage $(p=0.69)$. However, there was a statistically significant difference between the groups $(p=0.02)$ in reference to stress level. Table 2 illustrates the subjects' baseline characteristics.

\section{The effect of pulsed electromagnetic field on pain inten- sity and self-rated physical disability of low back pain}

The analysis showed a statistically significant improvement in pain intensity for the treatment group at weeks 3, 6, 9, and $13(p<0.05)$ (Table 3, Fig. 3). Pain intensity

Table 2. The description of the sample characteristics

\begin{tabular}{|c|c|c|c|}
\hline Characteristic & Treatment group $(\mathrm{n}=20)$ & Control group ( $\mathrm{n}=22)$ & $p$-value \\
\hline Age (yr) & $41.45 \pm 9.45$ & $42.61 \pm 9.69$ & 0.76 \\
\hline Body mass index $\left(\mathrm{kg} / \mathrm{m}^{2}\right)$ & $30.66 \pm 4.59$ & $31.13 \pm 7.57$ & 0.76 \\
\hline Gender & & & 0.77 \\
\hline Male & 10 & 12 & \\
\hline Female & 10 & 10 & \\
\hline Marital status & & & 0.93 \\
\hline Single & 2 & 3 & \\
\hline Married & 18 & 18 & \\
\hline Divorced & 0 & 1 & \\
\hline Employment & & & 0.36 \\
\hline Working & 15 & 18 & \\
\hline Not working & 5 & 4 & \\
\hline Pain intensity (0-10 NRS) & $5.70 \pm 1.97$ & $5.59 \pm 2.32$ & 0.87 \\
\hline Disability (RMDQ-24) & $8.95 \pm 3.64$ & $9.89 \pm 5.20$ & 0.51 \\
\hline Depression & $2.95 \pm 3.05$ & $5.41 \pm 5.97$ & 0.10 \\
\hline Anxiety & $2.95 \pm 3.36$ & $4.36 \pm 3.94$ & 0.06 \\
\hline Stress & $5.35 \pm 3.82$ & $9.09 \pm 5.97$ & 0.02 \\
\hline Pain medication use & & & 0.69 \\
\hline Yes & 7 & 9 & \\
\hline No & 13 & 13 & \\
\hline Duration of the low back pain (mo) & & & 0.50 \\
\hline $3-6$ & 3 & 3 & \\
\hline $6-12$ & 0 & 4 & \\
\hline$>12$ & 17 & 15 & \\
\hline
\end{tabular}

Values are presented as mean \pm standard deviation or number (\%). Bold type is considered statistically significant.

NRS, Numeric Rating Scale; RMDQ-24, Roland-Morris Disability Questionnaire. 
did not significantly improve at week 3 , but there was a significant improvement at weeks 6,9 , and $13(p<0.05)$ in the control group. The analysis of the self-rated physical disability scores for the treatment group revealed a statistically significant improvement in physical disability at weeks 3, 6, 9, and $13(p<0.05)$ (Table 3, Fig. 4). For the control group, there was no statistically significant difference in the self-rated physical disability at weeks 3 and 6 , but there was a significant improvement in weeks 9 and $13(p<0.05)$ (Table 3, Fig. 4).

The comparison between groups in pain intensity showed no statistically significant difference at all timepoint assessments (Table 3). However, the comparison between groups with respect to physical disability showed a significant improvement in the treatment group only at week $3(p<0.05)$ (Table 3$)$.

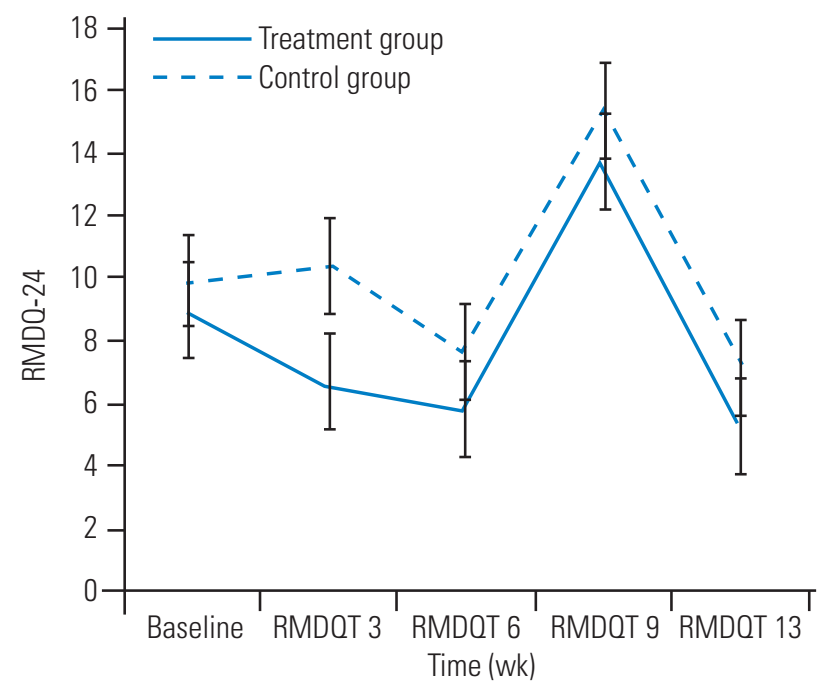

Fig. 4. The RMDO-24 score for the treatment and control groups. RMDQ-24, Roland-Morris Disability Questionnaire.
Fig. 3. The NPRS for the treatment and control groups. NPRS, Numeric Pain Rating Scale.

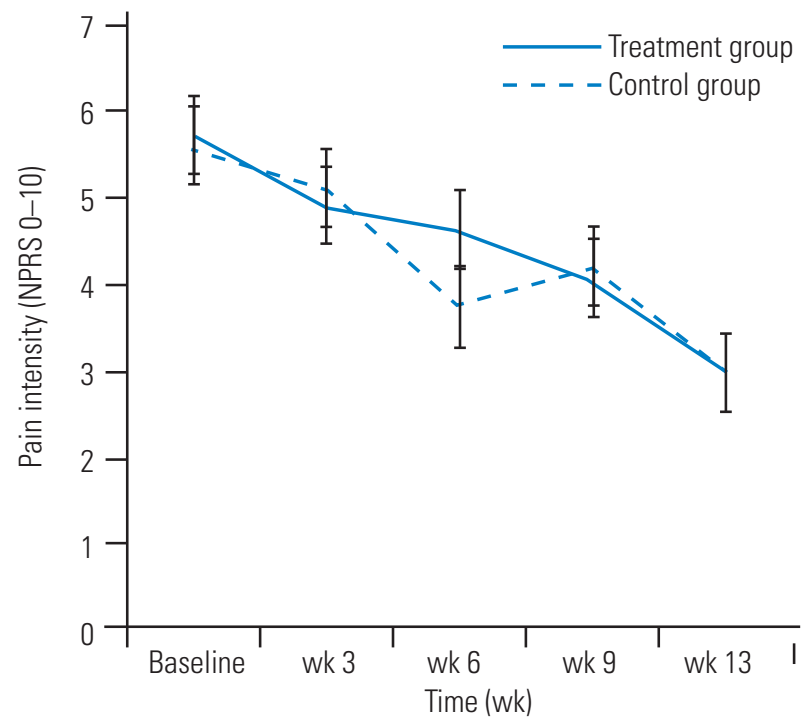

Table 3. The comparison of pain rating score and self-rated physical disability score between and within groups at different time-point assessments

\begin{tabular}{|c|c|c|c|c|c|}
\hline \multirow{2}{*}{ Period } & \multicolumn{2}{|c|}{ Treatment group $(\mathrm{n}=20)$} & \multicolumn{2}{|c|}{ Control group (n=22) } & \multirow{2}{*}{$\begin{array}{c}\text { Between groups } \\
p \text {-value }\end{array}$} \\
\hline & Mean $\pm S D$ & $p$-value & Mean \pm SD & $p$-value & \\
\hline \multicolumn{6}{|c|}{ Pain intensity (0-10 NRS) } \\
\hline Baseline & $5.70 \pm 1.97$ & & $5.59 \pm 2.32$ & & 0.87 \\
\hline wk 3 & $4.90 \pm 2.15$ & 0.04 & $5.09 \pm 2.40$ & 0.23 & 0.78 \\
\hline wk 6 & $4.60 \pm 2.18$ & 0.004 & $3.72 \pm 2.58$ & $<0.001$ & 0.24 \\
\hline wk 9 & $4.05 \pm 2.39$ & 0.002 & $4.18 \pm 3.01$ & 0.019 & 0.87 \\
\hline wk 13 & $2.95 \pm 2.16$ & $<0.001$ & $2.95 \pm 2.59$ & $<0.001$ & 0.99 \\
\hline \multicolumn{6}{|c|}{ Disability (RMDQ-24) } \\
\hline Baseline & $8.95 \pm 3.64$ & & $9.89 \pm 5.20$ & & 0.51 \\
\hline wk 3 & $6.65 \pm 4.13$ & 0.004 & $10.45 \pm 6.13$ & 0.56 & 0.02 \\
\hline wk 6 & $5.80 \pm 3.95$ & 0.002 & $7.72 \pm 6.58$ & 0.111 & 0.26 \\
\hline wk 9 & $13.75 \pm 2.84$ & $<0.001$ & $15.45 \pm 4.51$ & $<0.001$ & 0.10 \\
\hline wk 13 & $5.30 \pm 3.82$ & $<0.001$ & $7.18 \pm 6.93$ & 0.03 & 0.28 \\
\hline
\end{tabular}

SD, standard deviation; NRS, Numeric Rating Scale; RMDQ-24, Roland-Morris Disability Questionnaire. 
2. Effect of pulsed electromagnetic field on depression, psychological distress, sleep quality, and global perceived effect

There was a statistically significant improvement in the depression score in the treatment group only at week $13(p<0.05)$ (Table 4). For the control group, there was a statistically significant improvement in the depression score at weeks 6, 9, and $13(p<0.05)$ (Table 4$)$. The analysis of the stress scores for the treatment group revealed a statistically significant improvement at weeks 9 and 13 (Table 4). The control group had a statistically significant improvement in the stress score at all time-point assessments (Table 4). Furthermore, there was a statistically significant difference between the groups in relation to the stress scores at weeks 9 and $13(p<0.05)$, with a marginal improvement in the anxiety score between the groups at all time-point assessments (Table 4). However, no difference between the groups was found in sleep quality and the GPE.

Table 4. The comparison of the various scores between the treatment and control group

\begin{tabular}{|c|c|c|c|}
\hline Period & Treatment group (N=20) & Control group ( $\mathrm{N}=22$ ) & $p$-value ${ }^{* *}$ \\
\hline \multicolumn{4}{|c|}{ Pittsburgh Sleep Quality Index } \\
\hline Baseline & $2.25 \pm 0.71$ & $2.36 \pm 0.95$ & 0.66 \\
\hline wk 3 & $1.95 \pm 0.22$ & $2.23 \pm 0.75$ & 0.12 \\
\hline wk 6 & $2.05 \pm 0.75$ & $2.09 \pm 1.01$ & 0.88 \\
\hline wk 9 & $2.00 \pm 0.7$ & $2.05 \pm 0.95$ & 0.86 \\
\hline wk 13 & $1.65 \pm 0.58^{*}$ & $1.90 \pm 0.68$ & 0.19 \\
\hline \multicolumn{4}{|c|}{ Global perceived effect scale } \\
\hline Baseline & $-0.05 \pm 2.32$ & $0.14 \pm 2.71$ & 0.81 \\
\hline wk 3 & $0.40 \pm 1.75$ & $0.31 \pm 1.96$ & 0.88 \\
\hline wk 6 & $1.00 \pm 2.57$ & $1.86 \pm 2.05^{*}$ & 0.23 \\
\hline wk 9 & $1.50 \pm 2.46^{*}$ & $0.77 \pm 2.87$ & 0.38 \\
\hline wk 13 & $2.00 \pm 2.22^{*}$ & $1.82 \pm 2.44^{*}$ & 0.80 \\
\hline \multicolumn{4}{|l|}{ Depression } \\
\hline Baseline & $2.95 \pm 3.05$ & $5.41 \pm 5.97$ & 0.10 \\
\hline wk 3 & $2.25 \pm 2.97$ & $3.91 \pm 4.09$ & 0.14 \\
\hline wk 6 & $1.80 \pm 2.64$ & $3.95 \pm 4.81^{*}$ & 0.08 \\
\hline wk 9 & $1.35 \pm 3.01$ & $2.77 \pm 4.58^{*}$ & 0.24 \\
\hline wk 13 & $1.15 \pm 1.49^{*}$ & $3.40 \pm 5.27^{*}$ & 0.07 \\
\hline \multicolumn{4}{|l|}{ Anxiety } \\
\hline Baseline & $2.95 \pm 3.36$ & $4.36 \pm 3.94$ & 0.06 \\
\hline wk 3 & $2.30 \pm 3.26$ & $4.36 \pm 3.94$ & 0.07 \\
\hline wk 6 & $2.15 \pm 2.88$ & $4.36 \pm 4.49$ & 0.06 \\
\hline wk 9 & $1.70 \pm 3.40$ & $4.18 \pm 4.68$ & 0.05 \\
\hline wk 13 & $1.70 \pm 2.95$ & $3.77 \pm 4.24$ & 0.07 \\
\hline \multicolumn{4}{|l|}{ Stress } \\
\hline Baseline & $5.35 \pm 3.82$ & $9.09 \pm 5.97$ & 0.02 \\
\hline wk 3 & $4.70 \pm 3.86$ & $6.73 \pm 4.78^{*}$ & 0.14 \\
\hline wk 6 & $3.95 \pm 3.88$ & $5.68 \pm 4.28^{*}$ & 0.17 \\
\hline wk 9 & $2.80 \pm 3.18^{*}$ & $5.73 \pm 4.54^{*}$ & 0.02 \\
\hline wk 13 & $2.55 \pm 2.89^{*}$ & $5.77 \pm 5.53^{*}$ & 0.02 \\
\hline
\end{tabular}

Values are presented as mean \pm standard deviation or number (\%). Bold type is considered statistically significant.

${ }^{*}$ Within group $p$-value: significant level $<0.05$. ${ }^{* *}$ Between groups $p$-values $<0.05$. 


\section{Discussion}

Although PEMF resulted in a significant reduction in pain intensity and self-rated physical disability in patients with CLBP in the current study, it was not superior to therapeutic exercises given to the control group at the same period, which is similar to previously reported findings. Oke and Umebese [24] in 2013 reported a significant improvement in pain intensity and functional activity in the PEMF group but not in the standard medication group. A study by Gyulai et al. [21] in 2015 found that BEMER therapy reduced fatigue and pain intensity in the short term for CLBP patients. Moreover, Park et al. [25] in 2014 reported a significant decrease in pain intensity $(p<0.05)$ and functional disability $(p<0.01)$ in the PEMF group compared with the control group, but no significant difference in depression $(p=0.850)$ [25], similar to the present study. Omar et al. [10] in 2012 reported a significant difference between the PEMF treatment group and placebo group before and after application of PEMF in pain intensity, functional disability, and sleep quality ( $p=0.024, p<0.001$, and $p<0.001$, respectively) [10]. However, the present study revealed these differences were not significant. Elshiwi et al. [26] in 2018 reported a significant difference between the treatment group (PEMF therapy effects with $50 \mathrm{~Hz}$ frequency and low intensity of 20 Gauss) and the control group (conventional noninvasive treatment modalities) in the context of pain intensity (mean difference [MD], 1.52; 95\% confidence interval [CI], 0.34-3.35) and functional disability (MD, 8.14; 95\% CI, 6.5-9.96). Finally, Abdelhalim and Samhan [27] in 2018 reported a significant pain intensity reduction in the PEMF group $(p<0.05)$ compared with the control group after 3 months of treatment $(p>0.05)$.

On the contrary, other studies did not support our findings. For example, Harden et al. [28] in 2007 reported no significant difference in pain intensity throughout the treatment period $(p>0.05)$ between the treatment $(15$ millitesla PEMF group) and control groups. Similarly, Krammer et al. [29] in 2015 found that the PEMF group failed to demonstrate any significant improvement in pain intensity and functional disability $(p>0.05)$ over a 4 -week assessment period. Arneja et al. [9] in 2016 reported no significant improvement in the mental health total score of both the PEMF group and the control group ( $p=0.753$ and $p=0.447$, respectively). The literature suggests several factors might contribute to this discrepancy, for example, duration of treatment, parameters of the machine (frequency, pulse rate and width, magnetic flux density), and frequency of intervention, in addition to different followup periods.

\section{Recommendations}

Given these findings and considering the complexity and variety of patients with LBP, specific attention must be aimed at subgroups of patients with CLBP. CLBP patients should be screened for prognostic indicators by identifying modifiable risk factors (biomedical, psychological, and social) before initial decision-making. The current study findings support the notion that patients should be categorized into low, medium, and high-risk categories, and then treatment options should be customized [30].

\section{Study limitations}

Although there was no difference between the groups at baseline and the week 13 assessment point in analgesic intake, the study did not consider the dosage of analgesics that the patients were taking; different doses can affect the pain scores and, thus, act as a confounder. Therefore, future research must consider adjusting for these confounding variables by stratifying the patients according to dosage. In addition, the exercise therapy was not controlled between groups, which may explain the significant positive outcomes that were observed in the control group. Therefore, future research with a third arm of no exercise (sham only) would clarify this effect. Reliance on the participants for the measurement of outcome variables may have introduced participant bias. Lastly, baseline stress significantly differed between the PEMF and the control group, which raises the question whether the observed difference between the groups was due to confounding factors, such as bias in the questionnaire responses. Future researchers must ensure that the stress levels between the groups at baseline are similar.

\section{Conclusions}

No significant difference between the treatment and control groups was reported with respect to pain intensity, physical disability, sleep quality, and GPE, although there were significant improvements in the treatment group. This study has therefore concluded that PEMF therapy 
improves the outcome of CLBP patients. However, it is not superior to other treatment options. On the other hand, these findings make it obvious that CLBP is a complex condition, making it difficult to identify an effective treatment. Therefore, each patient needs to be assessed individually in order to tailor a suitable treatment plan.

\section{Conflict of Interest}

No potential conflict of interest relevant to this article was reported.

\section{Acknowledgments}

The authors acknowledge the department of physical therapy at King Fahad Specialist Hospital, Dammam for facilitating the data collection. we also acknowledge the study participants for their contribution. We also express our gratitude to all volunteers: Ms. Fatimah Adel Alkhameys, PT BSc, MSc, Ms. Eman Hassan Al-Abbas, PT BSc, Ms. Afnan Yahya AlZriri, PT BSc, Ms. Safia Khalid Al Arfaj PT BSc, and Ms. Mashael Farhan AL-Qahtani, PT BSc.

\section{Author Contributions}

Khalid Abdulkareem Alzayed: main investigator, data collection and analysis, report writing and publishing. Saad Mohammed Alsaadi: supervised data collection, managed data analysis and editing of the manuscript.

\section{References}

1. Freburger JK, Holmes GM, Agans RP, et al. The rising prevalence of chronic low back pain. Arch Intern Med 2009;169:251-8.

2. Vrbanic TS. Low back pain: from definition to diagnosis. Reumatizam 2011;58:105-7.

3. Stewart Williams J, Ng N, Peltzer K, et al. Risk factors and disability associated with low back pain in older adults in low- and middle-income countries: results from the WHO Study on Global AGEing and Adult Health (SAGE). PLoS One 2015;10:e0127880.

4. Maher C, Underwood M, Buchbinder R. Non-specific low back pain. Lancet 2017;389:736-47.

5. Chou R, Qaseem A, Snow V, et al. Diagnosis and treatment of low back pain: a joint clinical practice guideline from the American College of Physicians and the American Pain Society. Ann Intern Med 2007;147:478-91.

6. Yadollahpour A, Rashidi S. Electromagnetic field as a pain relieving modality: a review of the current literature. Int J Green Pharm 2017;11:S76-80.

7. Finegold L, Flamm BL. Magnet therapy. BMJ 2006;332:4.

8. Loo M. Integrative medicine for children. St. Louis (MO): Saunders/Elsevier; 2009.

9. Arneja AS, Kotowich A, Staley D, Summers R, Tappia PS. Electromagnetic fields in the treatment of chronic lower back pain in patients with degenerative disc disease. Future Sci OA 2016;2:FSO105.

10. Omar AS, Awadalla MA, El-Latif MA. Evaluation of pulsed electromagnetic field therapy in the management of patients with discogenic lumbar radiculopathy. Int J Rheum Dis 2012;15:e101-8.

11. Ferreira-Valente MA, Pais-Ribeiro JL, Jensen MP. Validity of four pain intensity rating scales. Pain 2011;152:2399-404.

12. Alghadir AH, Anwer S, Iqbal ZA. The psychometric properties of an Arabic numeric pain rating scale for measuring osteoarthritis knee pain. Disabil Rehabil 2016;38:2392-7.

13. Roland M, Fairbank J. The Roland-Morris Disability Questionnaire and the Oswestry Disability Questionnaire. Spine (Phila Pa 1976) 2000;25:3115-24.

14. Maki D, Rajab E, Watson PJ, Critchley DJ. Crosscultural translation, adaptation, and psychometric testing of the Roland-Morris disability questionnaire into modern standard Arabic. Spine (Phila Pa 1976) 2014;39:E1537-44.

15. Alsaadi SM, McAuley JH, Hush JM, et al. Detecting insomnia in patients with low back pain: accuracy of four self-report sleep measures. BMC Musculoskelet Disord 2013;14:196.

16. Cole JC, Motivala SJ, Buysse DJ, Oxman MN, Levin MJ, Irwin MR. Validation of a 3-factor scoring model for the Pittsburgh sleep quality index in older adults. Sleep 2006;29:112-6.

17. Suleiman KH, Yates BC, Berger AM, Pozehl B, Meza J. Translating the Pittsburgh Sleep Quality Index into Arabic. West J Nurs Res 2010;32:250-68.

18. Kamper SJ, Ostelo RW, Knol DL, Maher CG, de Vet HC, Hancock MJ. Global perceived effect scales provided reliable assessments of health transition in people with musculoskeletal disorders, but ratings 
are strongly influenced by current status. J Clin Epidemiol 2010;63:760-6.

19. Parkitny L, McAuley J. The Depression Anxiety Stress Scale (DASS). J Physiother 2010;56:204.

20. Moussa MT, Lovibond P, Laube R, Megahead HA. Psychometric properties of an Arabic version of the depression anxiety stress scales (DASS). Res Soc Work Pract 2017;27:375-86.

21. Gyulai F, Raba K, Baranyai I, Berkes E, Bender T. BEMER therapy combined with physiotherapy in patients with musculoskeletal diseases: a randomised, controlled double blind follow-up pilot study. Evid Based Complement Alternat Med 2015;2015:245742.

22. Airaksinen O, Brox JI, Cedraschi C, et al. Chapter 4: European guidelines for the management of chronic nonspecific low back pain. Eur Spine J 2006;15 Suppl 2:S192-300.

23. Hayden JA, van Tulder MW, Malmivaara A, Koes BW. Exercise therapy for treatment of non-specific low back pain. Cochrane Database Syst Rev 2005;(3):CD000335.

24. Oke KI, Umebese PF. Evaluation of the efficacy of pulsed electromagnetic therapy in the treatment of back pain: a randomized controlled trial in a tertiary hospital in Nigeria. West Indian Med J 2013;62:2059.

25. Park WH, Sun SH, Lee SG, et al. Effect of pulsed electromagnetic field treatment on alleviation of lumbar myalgia: a single center, randomized, doubleblind, sham-controlled pilot trial study. J Magn 2014;19:161-9.

26. Elshiwi AM, Hamada HA, Mosaad D, Ragab IM, Koura GM, Alrawaili SM. Effect of pulsed electromagnetic field on nonspecific low back pain patients: a randomized controlled trial. Braz J Phys Ther 2019;23:244-9.

27. Abdelhalim NM, Samhan AF. Long term effects of low-frequency magnetic field therapy in treatment patients with low back pain. Int J Med Res Health Sci 2018;7:108-13.

28. Harden RN, Remble TA, Houle TT, Long JF, Markov MS, Gallizzi MA. Prospective, randomized, singleblind, sham treatment-controlled study of the safety and efficacy of an electromagnetic field device for the treatment of chronic low back pain: a pilot study. Pain Pract 2007;7:248-55.

29. Krammer A, Horton S, Tumilty S. Pulsed electromagnetic energy as an adjunct to physiotherapy for the treatment of acute low back pain: a randomised controlled trial. NZ J Physiother 2015;43:16-22.

30. Robinson HS, Dagfinrud H. Reliability and screening ability of the StarT back screening tool in patients with low back pain in physiotherapy practice, a cohort study. BMC Musculoskelet Disord 2017;18:232. 